Quality of life

\section{Why quality of life should matter to occupational health researchers}

\section{P D Blanc}

\section{Comment on the article by Pattani et al (see page 572)}

T he article by Dr Pattani and colleagues in this issue of $O E M$ takes on the very difficult and important question of quality of life in relation to working life. ${ }^{1}$ Quality of life-insofar as it relates to health status-has become a central concern to a wide range of medical investigators. Not only is quality of life a major variable to be considered in any study of the natural history of disease processes, it is also a critical outcome that must be gauged when assessing the efficacy of an intervention intended to modify that natural history.

The term "quality of life" is widely used, but often loosely applied. Health related quality of life, as a construct, is meant to capture the experience of health and disease from the viewpoint of the individual. Does the person in question perceive being in a state of health, self-graded along a spectrum from poor to excellent? Is one severely limited in performing routine physical activities or without any such limitation? To what extent are mood, outlook, or emotional spirits bright or dim? Because these generic assessments are fairly broad by their very nature, quality of life assessment tools have been refined for greater disease specific definition of the problem. This allows identification of constellations of particular issues that may be clustered more closely to certain health conditions, for example asthma or musculoskeletal disorders. Thus an asthma related, disease specific quality of life scale includes questions particularly pertinent to the perception of breathing difficulties and their impact on the life of the person experiencing this disorder.

In contrast to health care research generally, occupational medical research has been fairly late in coming to consider quality of life as a focus of investigation, either by using generic measures (such as the Short Form 36 reported by Pattani et al) or disease specific questionnaire batteries. This may be attributable, at least in part, to a more concrete world view, wherein the outcomes of principal concern to the occupational researcher have often been work related disabling or fatal injury or disease, manifest by frank job loss or lost work time. There may also be an element of class based assumptions coming into play; that is to say, a presumption that for the industrial or agricultural worker, "quality of life" is a vague luxury, not comparable to the bread-and-butter priority of traditional safety and health concerns.

There is, however, an even more fundamental set of issues involved when considering quality of life in relation to employment status in health and disease. To the extent that meaningful employment is an essential component of daily life, providing not only income, but also a sense of engagement, role identification, and physical and mental stimulation, work and quality of life are very difficult to disentangle analytically. Is a decrement in one's health related quality of life reflective of an injury or a disease process leading to work loss, or is work loss itself a harbinger of poorer quality of life and a risk factor for disease?

A related debate over disability and potential income replacement countered the theoretical argument that "relatively" generous social insurance schemes promote increased disability rates with convincing data that the nature of work itself is what drives persons to leave the labour force due to health limitations. ${ }^{2}$ Even more germane to the interrelationships among meaningful work, income, and health status, was a study of unionised printers whose professional duties were made redundant by changing technology. The printers formally retained their employment by contract right. This protected their salary, but they were forced to come to the workplace every day with no job to actually perform, a change in the nature of work temporally linked to worsening health status. ${ }^{3}$

Pattani and colleagues can get at part of the question of health related quality of life and work status. They show that work disability is related to poor quality of life and that those with poorer quality of life at baseline (at the time of leaving work) are less likely to return to work, even part-time. The association between work status and the physical health component of quality of life was not altered after taking other co-factors into account (including job type and the nature of the medical condition involved). In contrast, the mental component of quality of life emerged as a potent predictive factor only after adjusting for the same co-factors, suggesting that confounding relationships exist among these.

There was also an association between return to work and improved quality of life over baseline. Here we cannot say which came first and either order of events is plausible. Despite the longitudinal design of the study, it cannot tease out the sequential relationships among health status, work status, and perceived quality of life. Perhaps this is less important than the central observation underlying this report: that quality of life and working life are linked together and that both, therefore, must be of concern to the occupational health researcher

Occup Environ Med 2004;61:571.

doi: 10.1136/oem.2004.013888

Correspondence to: Dr P D Blanc, 350

Parnassus Avenue, Suite 609, San Francisco, CA 94117, USA; blancp@itsa.ucsf.edu

\section{REFERENCES}

1 Pattani S, Constantinovici N, Williams S Predictors of re-employment and quality of life in NHS staff one year after early retirement because of ill health; a national prospective study. Occup Environ Med 2004;61:572-6.

2 Yelin EH. Disability and the displaced worker New Brunswick, NJ: Rutgers University Press, 1992

3 Michaels DM. Technological change, deskilling and heart disease among skilled blue collar workers: what becomes of the broken-hearted. Doctoral dissertation. New York: Columbia University, 1987. 